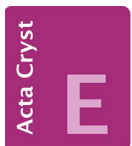

CRYSTALLOGRAPHIC COMMUNICATIONS

ISSN 2056-9890

Received 4 April 2015

Accepted 21 April 2015

Edited by I. D. Brown, McMaster University, Canada

Keywords: crystal structure; transition metal phosphates; solid-state reaction synthesis; $\mathrm{Na}_{2} \mathrm{Co}_{2} \mathrm{Fe}\left(\mathrm{PO}_{4}\right)_{3}$; alluaudite-like structure

CCDC reference: 1060932 Supporting information: this article has supporting information at journals.iucr.org/e

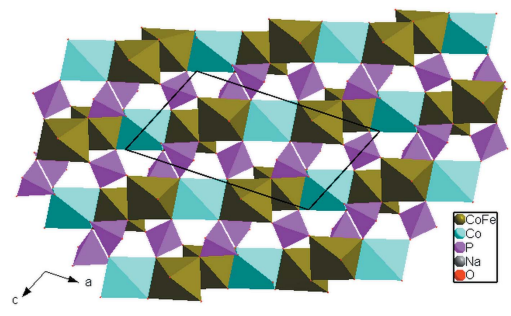

OPEN $\odot$ ACCESS

\section{Crystal structure of disodium dicobalt(II) iron(III) tris(orthophosphate) with an alluaudite-like structure}

\author{
Adam Bouraima, ${ }^{a, b *}$ Abderrazzak Assani, ${ }^{a}$ Mohamed Saadi, ${ }^{a}$ Thomas Makani ${ }^{b}$ and \\ Lahcen El Ammari ${ }^{a}$
}

aLaboratoire de Chimie du Solide Appliquée, Faculté des Sciences, Université Mohammed V, Avenue Ibn Battouta, BP 1014, Rabat, Morocco, and ' ${ }^{\mathbf{b}}$ Département de chimie, Faculté des Sciences, Université des Sciences et Techniques de Masuku, BP 943, Franceville, Gabon. *Correspondence e-mail: adam_bouraima@yahoo.fr

The title compound, $\mathrm{Na}_{2} \mathrm{Co}_{2} \mathrm{Fe}\left(\mathrm{PO}_{4}\right)_{3}$, was synthesized by a solid-state reaction. This new stoichiometric phase crystallizes in an alluaudite-like structure. In this structure, all atoms are in general positions except for four atoms which are located at the special positions of the $C 2 / c$ space group. One Co atom, one $\mathrm{P}$ and one $\mathrm{Na}$ atom are all located on Wyckoff position $4 e(2)$, while the second $\mathrm{Na}$ atom is located on an inversion centre $4 a(\overline{1})$. The other Co and Fe atoms occupy a general position with a statistical distribution. The open framework results from $\left[(\mathrm{Co}, \mathrm{Fe})_{2} \mathrm{O}_{10}\right]$ units of edge-sharing $\left[(\mathrm{Co}, \mathrm{Fe}) \mathrm{O}_{6}\right]$ octahedra, which alternate

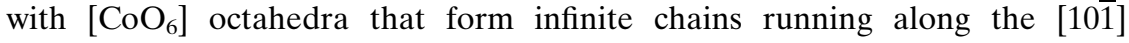
direction. These chains are linked together through $\mathrm{PO}_{4}$ tetrahedra by the sharing of vertices so as to build layers perpendicular to [010]. The threedimensional framework is accomplished by the stacking of these layers, leading to the formation of two types of tunnels parallel to [010] in which the $\mathrm{Na}^{+}$cations are located, each cation being surrounded by eight $\mathrm{O}$ atoms.

\section{Chemical context}

A particular focus of ours concerns compounds with alluaudite-type structures, and we set the objective of synthesising new transition-metal-based phosphates within the well-known alluaudite family. We are interested in this because transitionmetal phosphates are of great interest with applications in several fields. Compounds belonging to the large structural family of derivatives (Moore, 1971) have been of continuing interest due to their structural properties, such as their openframework architecture and their physical properties. Moreover, the flexibility of the alluaudite structure will, no doubt, permit the use of alluaudite-type phosphates for practical applications, such as corrosion inhibition, passivation of metal surfaces and catalysis (Korzenski et al., 1999). These materials abound in magnetic properties of metallic phosphate. Transition metals can play an important role in microporous skeletons by supplying an active catalytic site keeping the selectivity of frames (Weil et al., 2009). Metallic phosphates present a multitude of structural wealth which are the object of studies of catalysts (Viter \& Nagornyi, 2009; Gao \& Gao, 2005), ion exchange (Clearfield, 1988) and the positive electrode in lithium and sodium batteries (Trad et al., 2010). As a result of the presence of channels parallel to [100], alluauditetype compounds exhibit electronic and/or ionic conductivity, as has been shown by Warner et al. (1993). In this context, we have explored $A_{2} \mathrm{O}-M \mathrm{O}-\mathrm{P}_{2} \mathrm{O}_{5}$ systems, where $A$ is a monovalent cation and $M$ a divalent cation. A new alluaudite 


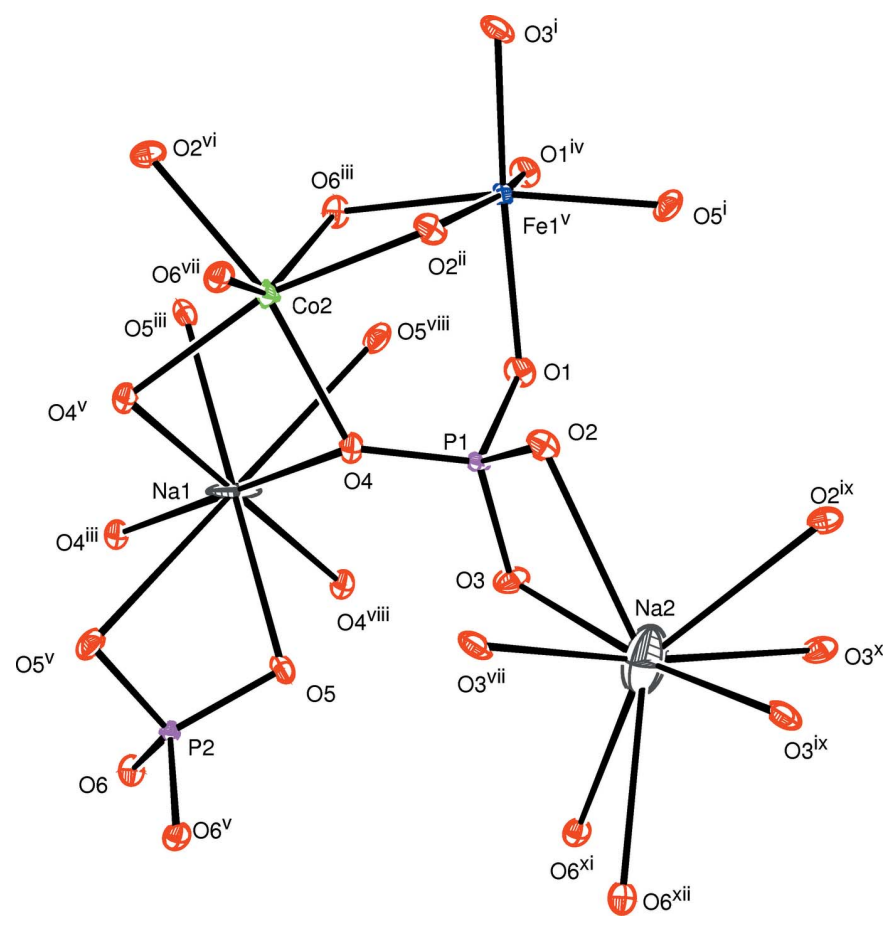

Figure 1

The principal building units in the structure of the title compound. Displacement ellipsoids are drawn at the $50 \%$ probability level. Symmetry codes: (i) $-x+\frac{3}{2}, y+\frac{1}{2},-z+\frac{1}{2}$; (ii) $-x+\frac{3}{2},-y+\frac{3}{2},-z+1$; (iii) $-x+1,-y+1,-z$; (iv) $-x+\frac{3}{2},-y+\frac{3}{2},-z$; (v) $-x+1, y,-z+\frac{1}{2}$; (vi) $x-\frac{1}{2},-y+\frac{3}{2}, z-\frac{1}{2}$; (vii) $x,-y+1, z+\frac{1}{2}$; (viii) $x,-y+1, z-\frac{1}{2}$; (ix) $-x+2$, $y,-z+\frac{3}{2}$; (x) $-x+2,-y+1,-z+1$; (xi) $x+\frac{1}{2},-y+\frac{1}{2}, z+\frac{1}{2}$; (xii) $-x+\frac{3}{2}$, $-y+\frac{1}{2},-z+1$.

structure of formula $\mathrm{Na}_{2} \mathrm{Co}_{2} \mathrm{Fe}\left(\mathrm{PO}_{4}\right)_{3}$ was synthesized by solid-state reaction. During our investigation of these systems, we characterized the following compounds: $\mathrm{AgMg}_{3}\left(\mathrm{PO}_{4}\right)$ $\left(\mathrm{HPO}_{4}\right)_{2}$ (Assani et al., 2011a), $\mathrm{Ag}_{2} \mathrm{Ni}_{3}\left(\mathrm{HPO}_{4}\right)\left(\mathrm{PO}_{4}\right)_{2}$ (Assani et al., 2011b) and $\mathrm{Na}_{2} \mathrm{Ni}_{2} \mathrm{Fe}\left(\mathrm{PO}_{4}\right)_{3}$ (Essehli et al., 2011). The present paper reports the solid-state synthesis and characterization of a new transition-metal phosphate, namely, $\mathrm{Na}_{2} \mathrm{Co}_{2} \mathrm{Fe}\left(\mathrm{PO}_{4}\right)_{3}$.

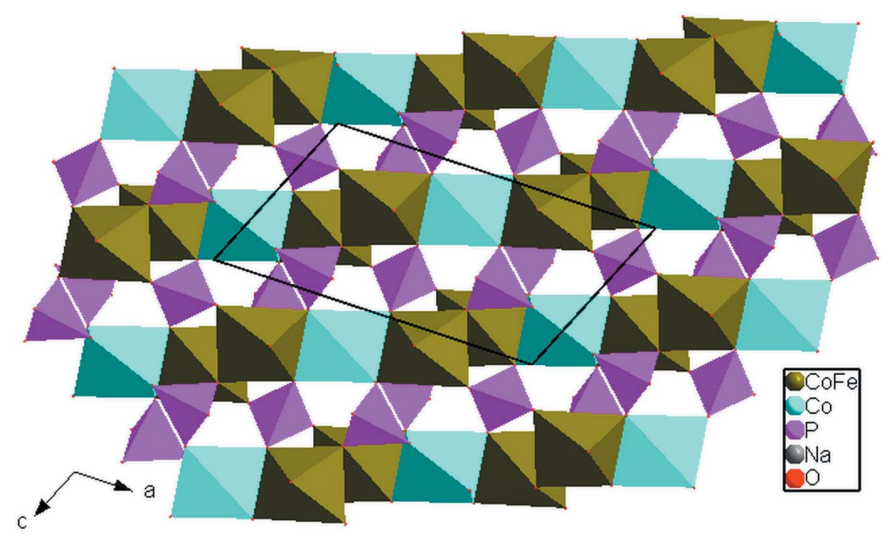

Figure 2

A layer perpendicular to the $b$ axis, resulting from the chains connected via the vertices of the $\mathrm{PO}_{4}$ tetrahedra.

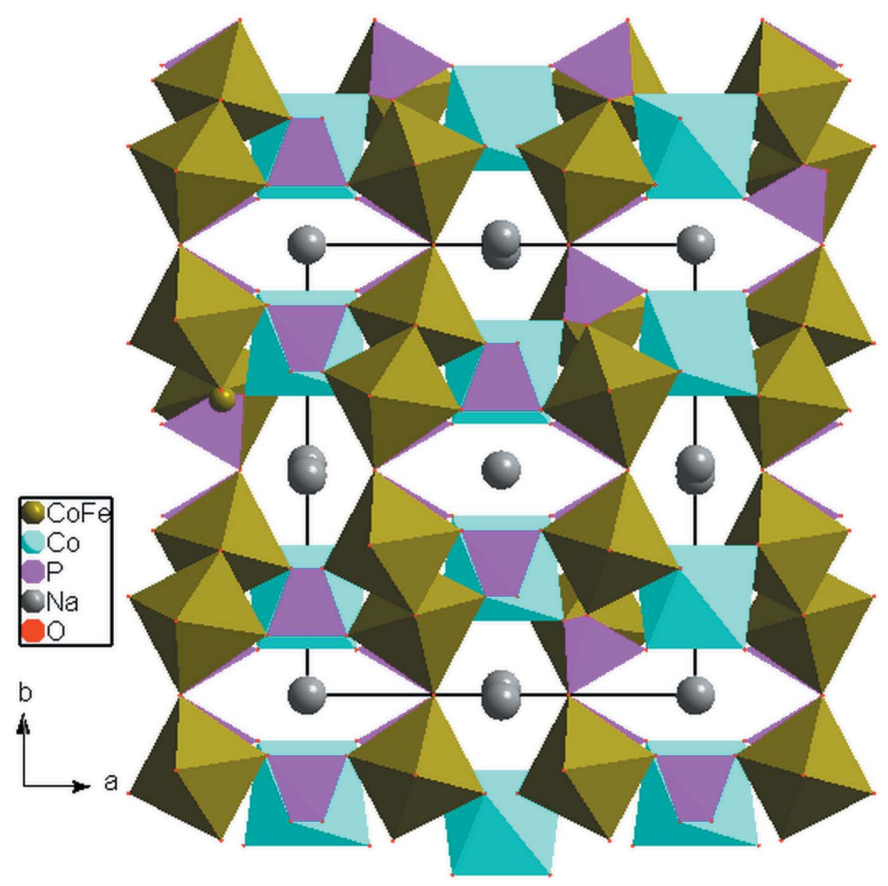

Figure 3

Polyhedral representation of $\mathrm{Na}_{2} \mathrm{Co}_{2} \mathrm{Fe}\left(\mathrm{PO}_{4}\right)_{3}$ showing the tunnels running along the [001] direction.

\section{Structural commentary}

In the refinement of the first model of this structure, we placed the $\mathrm{Fe}$ atom in Wyckoff position $4 e$ and $\mathrm{Co}$ in the general position $8 f$. The results of the refinement of this model are acceptable if we disregard the high weight values. However, bond-valence-sum calculations (Brown \& Altermatt, 1985) are not in favor of this model and, consequently, the examination of all possible models led to the best one in which half of the $\mathrm{Co}, \mathrm{Na}$, and $\mathrm{P}$ atoms are in Wyckoff position $4 e$, and the second $\mathrm{Na}$ atom is in position $4 a$ of the $C 2 / c$ space group, the remaining $\mathrm{Co}$ and $\mathrm{Fe}$ fulfilling the $8 f$ site. In this case, bondvalence-sum calculations for $\mathrm{Co}_{2}{ }^{2+}, \mathrm{Co}^{2+}, \mathrm{Fe}^{2+}, \mathrm{Na}^{+}, \mathrm{Na}^{+}$, $\mathrm{P}^{5+}$ and $\mathrm{P} 2^{5+}$ ions are as expected, viz 1.78, 2.02, 2.81, 1.25, $0.94,4.98$ and 4.99 valence units, respectively.

The new phase of formula $\mathrm{Na}_{2} \mathrm{Co}_{2} \mathrm{Fe}\left(\mathrm{PO}_{4}\right)_{3}$ crystallizes in the alluaudite type. The structure of this compound is built up from two edge-sharing $\left[(\mathrm{Co}, \mathrm{Fe}) \mathrm{O}_{6}\right]$ octahedra, leading to the formation of $\left[(\mathrm{Co}, \mathrm{Fe})_{2} \mathrm{O}_{10}\right]$ dimers that are connected by a common edge to $\left[\mathrm{CoO}_{6}\right]$ octahedra, as shown in Fig.1. The linkage of alternating $\left[\mathrm{CoO}_{6}\right]$ and $\left[(\mathrm{Co}, \mathrm{Fe})_{2} \mathrm{O}_{10}\right]$ octahedra

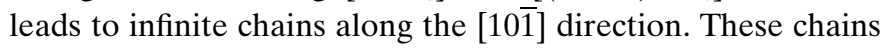
held together via the vertices of the $\mathrm{PO}_{4}$ tetrahedra in such a way as to build layers perpendicular to [010] (Fig. 2). The junction of different octahedra by common vertices of $\mathrm{PO}_{4}$ tetrahedra form an open three-dimensional framework that delimits two types of tunnels parallel to [100] and [001] accommodating the $\mathrm{Na}^{+}$cations, as shown in Fig. 3. In the tunnels, each sodium atom is surrounded by eight oxygen atoms with $\mathrm{Na} 1-\mathrm{O}$ and $\mathrm{Na} 2-\mathrm{O}$ bond lengths varying between 2.2895 (9) and 2.8754 (10) $\AA$ ) and between 2.3940 (9) and 2.8513 (16) $\AA$, respectively. 


\section{Synthesis and crystallization}

$\mathrm{Na}_{2} \mathrm{Co}_{2} \mathrm{Fe}\left(\mathrm{PO}_{4}\right)_{3}$ was synthesized by a solid-state reaction by mixing the precursors of sodium $\left(\mathrm{Na}_{2} \mathrm{CO}_{3}\right)$, cobalt $\left(\mathrm{CoCO}_{3}\right)$, iron $\left(\mathrm{Fe}_{2} \mathrm{O}_{3}\right)$ and phosphoric acid $85 \%$ wt. The various precursors were taken in the molar ratio Na:Co:Fe:P = 2:2:1:3.

After different heat treatments in a platinum crucible up to $873 \mathrm{~K}$, the reaction mixture was heated to the melting point of $1000 \mathrm{~K}$. The molten product was then cooled to room temperature at a rate of $5 \mathrm{~K} \mathrm{~h}^{-1}$. The resulting product contained brown crystals of a suitable size for the X-ray diffraction study.

\section{Refinement}

Crystal data, data collection and structure refinement details are summarized in Table 1 . The same $x, y$ and $z$ parameters and anisotropic displacement parameters are used for Co1 and Fe1 sharing the same site. Three reflections, (042), (110) and $(\overline{2} 42)$, probably affected by the beam-stop, were removed during the last refinement cycle. The highest peak and the deepest hole in the final Fourier map are at $0.40 \AA$ and $0.42 \AA$ from $\mathrm{Na} 1$ and $\mathrm{Na} 2$, respectively.

\section{Acknowledgements}

The authors thank the Unit of Support for Technical and Scientific Research (UATRS, CNRST) for the X-ray measurements and Mohammed V University, Rabat, Morocco, for financial support.

\section{References}

Assani, A., El Ammari, L., Zriouil, M. \& Saadi, M. (2011b). Acta Cryst. E67, i40.

Assani, A., Saadi, M., Zriouil, M. \& El Ammari, L. (2011a). Acta Cryst. E67, i5.

Brandenburg, K. (2006). DIAMOND. Crystal Impact GbR, Bonn, Germany.

Brown, I. D. \& Altermatt, D. (1985). Acta Cryst. B41, 244-247.

Bruker (2009). APEX2, SAINT and SADABS. Bruker AXS Inc., Madison, Wisconsin, USA.

Clearfield, A. (1988). Chem. Rev. 88, 125-148.

Essehli, R., El Bali, B., Benmokhtar, S., Bouziane, K., Manoun, B., Abdalslam, M. A. \& Ehrenberg, H. (2011). J. Alloys Compd. 509, 1163-1171.
Table 1

Experimental details.

\begin{tabular}{|c|c|}
\hline \multicolumn{2}{|l|}{ Crystal data } \\
\hline Chemical formula & $\mathrm{Na}_{2} \mathrm{Co}_{2} \mathrm{Fe}\left(\mathrm{PO}_{4}\right)_{3}$ \\
\hline$M_{\mathrm{r}}$ & 504.60 \\
\hline Crystal system, space group & Monoclinic, $C 2 / c$ \\
\hline Temperature $(\mathrm{K})$ & 296 \\
\hline$a, b, c(\AA)$ & $11.7106(6), 12.4083(7), 6.4285(3)$ \\
\hline$\beta\left(^{\circ}\right)$ & $113.959(2)$ \\
\hline$V\left(\AA^{3}\right)$ & $853.63(8)$ \\
\hline$Z$ & 4 \\
\hline Radiation type & Mo $K \alpha$ \\
\hline$\mu\left(\mathrm{mm}^{-1}\right)$ & 6.26 \\
\hline Crystal size $(\mathrm{mm})$ & $0.31 \times 0.25 \times 0.19$ \\
\hline \multicolumn{2}{|l|}{ Data collection } \\
\hline Diffractometer & Bruker X8 APEX \\
\hline Absorption correction & $\begin{array}{l}\text { Multi-scan ( } S A D A B S ; \text { Bruker, } \\
\text { 2009) }\end{array}$ \\
\hline$T_{\min }, T_{\max }$ & $0.504,0.748$ \\
\hline $\begin{array}{l}\text { No. of measured, independent and } \\
\text { observed }[I>2 \sigma(I)] \text { reflections }\end{array}$ & $15289,1882,1807$ \\
\hline$R_{\text {int }}$ & 0.030 \\
\hline$(\sin \theta / \lambda)_{\max }\left(\AA^{-1}\right)$ & 0.806 \\
\hline \multicolumn{2}{|l|}{ Refinement } \\
\hline$R\left[F^{2}>2 \sigma\left(F^{2}\right)\right], w R\left(F^{2}\right), S$ & $0.016,0.046,1.10$ \\
\hline No. of reflections & 1879 \\
\hline No. of parameters & 95 \\
\hline$\Delta \rho_{\max }, \Delta \rho_{\min }\left(\mathrm{e} \AA^{-3}\right)$ & $0.70,-0.92$ \\
\hline
\end{tabular}

Computer programs: APEX2 and SAINT (Bruker, 2009), SHELXS97 and SHELXL97 (Sheldrick, 2008), ORTEP-3 for Windows (Farrugia, 2012), DIAMOND (Brandenburg, 2006) and publCIF (Westrip, 2010).

Farrugia, L. J. (2012). J. Appl. Cryst. 45, 849-854.

Gao, D. \& Gao, Q. (2005). Micropor. Mesopor. Mater. 85, 365-373.

Korzenski, M. B., Kolis, J. W. \& Long, G. J. (1999). J. Solid State Chem. 147, 390-398.

Moore, P. B. (1971). Am. Mineral. 56, 1955-1975.

Sheldrick, G. M. (2008). Acta Cryst. A64, 112-122.

Trad, K., Carlier, D., Croguennec, L., Wattiaux, A., Ben Amara, M. \& Delmas, C. (2010). Chem. Mater. 22, 5554-5562.

Viter, V. N. \& Nagornyi, P. G. (2009). Russ. J. Appl. Chem. 82, 935939.

Warner, T. E., Milius, W. \& Maier, J. (1993). J. Solid State Chem. 106, 301-309.

Weil, M., Đorđević, T., Lengauer, C. L. \& Kolitsch, U. (2009). Solid State Sci. 11, 2111-2117.

Westrip, S. P. (2010). J. Appl. Cryst. 43, 920-925. 


\section{supporting information}

Acta Cryst. (2015). E71, 558-560 [https://doi.org/10.1107/S2056989015007926]

Crystal structure of disodium dicobalt(II) iron(III) tris(orthophosphate) with an alluaudite-like structure

Adam Bouraima, Abderrazzak Assani, Mohamed Saadi, Thomas Makani and Lahcen El Ammari

Computing details

Data collection: APEX2 (Bruker, 2009); cell refinement: SAINT (Bruker, 2009); data reduction: SAINT (Bruker, 2009); program(s) used to solve structure: SHELXS97 (Sheldrick, 2008); program(s) used to refine structure: SHELXL97 (Sheldrick, 2008); molecular graphics: ORTEP-3 for Windows (Farrugia, 2012) and DIAMOND (Brandenburg, 2006); software used to prepare material for publication: publCIF (Westrip, 2010).

Disodium dicobalt(II) iron(III) tris(orthophosphate)

Crystal data

$\mathrm{Na}_{2} \mathrm{Co}_{2} \mathrm{Fe}\left(\mathrm{PO}_{4}\right)_{3}$

$M_{r}=504.60$

Monoclinic, $C 2 / c$

Hall symbol: $-\mathrm{c} 2 \mathrm{yc}$

$a=11.7106(6) \AA$

$b=12.4083(7) \AA$

$c=6.4285(3) \AA$

$\beta=113.959(2)^{\circ}$

$V=853.63(8) \AA^{3}$

$Z=4$

$F(000)=972$

$D_{\mathrm{x}}=3.926 \mathrm{Mg} \mathrm{m}^{-3}$

Mo $K \alpha$ radiation, $\lambda=0.71073 \AA$

Cell parameters from 1882 reflections

$\theta=2.5-34.9^{\circ}$

$\mu=6.26 \mathrm{~mm}^{-1}$

$T=296 \mathrm{~K}$

Block, brown

$0.31 \times 0.25 \times 0.19 \mathrm{~mm}$

\section{Data collection}

\section{Bruker X8 APEX}

diffractometer

Radiation source: fine-focus sealed tube

Graphite monochromator

$\varphi$ and $\omega$ scans

Absorption correction: multi-scan

(SADABS; Bruker, 2009)

$T_{\text {min }}=0.504, T_{\max }=0.748$

15289 measured reflections

1882 independent reflections

1807 reflections with $I>2 \sigma(I)$

$R_{\text {int }}=0.030$

$\theta_{\text {max }}=34.9^{\circ}, \theta_{\min }=2.5^{\circ}$

$h=-18 \rightarrow 18$

$k=-20 \rightarrow 20$

$l=-9 \rightarrow 10$

Refinement

Refinement on $F^{2}$

Least-squares matrix: full

$R\left[F^{2}>2 \sigma\left(F^{2}\right)\right]=0.016$

$w R\left(F^{2}\right)=0.046$

$S=1.10$

1879 reflections

95 parameters

0 restraints

Primary atom site location: structure-invariant direct methods

Secondary atom site location: difference Fourier map

$w=1 /\left[\sigma^{2}\left(F_{\mathrm{o}}^{2}\right)+(0.026 P)^{2}+1.033 P\right]$

where $P=\left(F_{\mathrm{o}}^{2}+2 F_{\mathrm{c}}^{2}\right) / 3$

$(\Delta / \sigma)_{\max }=0.002$

$\Delta \rho_{\max }=0.70 \mathrm{e} \AA^{-3}$ 
$\Delta \rho_{\min }=-0.92$ e $\AA^{-3}$
Extinction correction: SHELXL97 (Sheldrick, 2008), $\mathrm{Fc}^{*}=\mathrm{kFc}\left[1+0.001 \mathrm{xFc}^{2} \lambda^{3} / \sin (2 \theta)\right]^{-1 / 4}$

Extinction coefficient: 0.0049 (3)

Special details

Geometry. All esds (except the esd in the dihedral angle between two 1.s. planes) are estimated using the full covariance matrix. The cell esds are taken into account individually in the estimation of esds in distances, angles and torsion angles; correlations between esds in cell parameters are only used when they are defined by crystal symmetry. An approximate (isotropic) treatment of cell esds is used for estimating esds involving l.s. planes.

Refinement. Refinement of $F^{2}$ against all reflections. The weighted $R$-factor $w R$ and goodness of fit $S$ are based on $F^{2}$, conventional $R$-factors $R$ are based on $F$, with $F$ set to zero for negative $F^{2}$. The threshold expression of $F^{2}>\sigma\left(F^{2}\right)$ is used only for calculating $R$-factors (gt) etc. and is not relevant to the choice of reflections for refinement. $R$-factors based on $F^{2}$ are statistically about twice as large as those based on $F$, and $R$ - factors based on all data will be even larger.

Fractional atomic coordinates and isotropic or equivalent isotropic displacement parameters $\left(\AA^{2}\right)$

\begin{tabular}{llllll}
\hline & $x$ & $y$ & $z$ & $U_{\text {iso }} / U_{\text {eq }}$ & Occ. $(<1)$ \\
\hline Fe1 & $0.717910(14)$ & $0.842242(12)$ & $0.13094(2)$ & $0.00487(5)$ & 0.50 \\
Co1 & $0.717910(14)$ & $0.842242(12)$ & $0.13094(2)$ & $0.00487(5)$ & 0.50 \\
Co2 & 0.5000 & $0.731171(18)$ & 0.2500 & $0.00645(5)$ & \\
P1 & $0.76483(3)$ & $0.60996(2)$ & $0.37608(4)$ & $0.00406(6)$ & \\
P2 & 0.5000 & $0.29046(3)$ & 0.2500 & $0.00393(7)$ & \\
Na1 & 0.5000 & 0.5000 & 0.0000 & $0.01623(17)$ & \\
Na2 & 1.0000 & $0.48710(12)$ & 0.7500 & $0.0343(3)$ & \\
O1 & $0.77862(8)$ & $0.67773(7)$ & $0.18650(14)$ & $0.00805(14)$ & \\
O2 & $0.83828(8)$ & $0.66574(7)$ & $0.60820(14)$ & $0.00755(14)$ & \\
O3 & $0.82670(9)$ & $0.49990(7)$ & $0.38761(16)$ & $0.00954(15)$ & \\
O4 & $0.62676(8)$ & $0.60150(7)$ & $0.32737(15)$ & $0.00871(14)$ & \\
O5 & $0.60276(8)$ & $0.36657(7)$ & $0.25279(15)$ & $0.00938(15)$ & \\
O6 & $0.45930(8)$ & $0.21880(7)$ & $0.03503(13)$ & $0.00683(13)$ & \\
& & & & & \\
\end{tabular}

Atomic displacement parameters $\left(\AA^{2}\right)$

\begin{tabular}{lllllll}
\hline & $U^{11}$ & $U^{22}$ & $U^{33}$ & $U^{12}$ & $U^{13}$ & $U^{23}$ \\
\hline Fe1 & $0.00449(7)$ & $0.00506(7)$ & $0.00514(7)$ & $-0.00034(4)$ & $0.00203(5)$ & $-0.00037(4)$ \\
Co1 & $0.00449(7)$ & $0.00506(7)$ & $0.00514(7)$ & $-0.00034(4)$ & $0.00203(5)$ & $-0.00037(4)$ \\
Co2 & $0.00659(9)$ & $0.00646(9)$ & $0.00728(9)$ & 0.000 & $0.00381(7)$ & 0.000 \\
P1 & $0.00506(11)$ & $0.00343(11)$ & $0.00348(10)$ & $-0.00004(8)$ & $0.00150(8)$ & $-0.00003(7)$ \\
P2 & $0.00357(15)$ & $0.00423(15)$ & $0.00352(14)$ & 0.000 & $0.00094(11)$ & 0.000 \\
Na1 & $0.0262(4)$ & $0.0032(3)$ & $0.0068(3)$ & $0.0017(3)$ & $-0.0061(3)$ & $0.0008(2)$ \\
Na2 & $0.0187(5)$ & $0.0569(8)$ & $0.0218(5)$ & 0.000 & $0.0026(4)$ & 0.000 \\
O1 & $0.0094(3)$ & $0.0097(3)$ & $0.0052(3)$ & $-0.0011(3)$ & $0.0031(3)$ & $0.0014(3)$ \\
O2 & $0.0097(3)$ & $0.0073(3)$ & $0.0049(3)$ & $-0.0018(3)$ & $0.0021(3)$ & $-0.0015(2)$ \\
O3 & $0.0113(4)$ & $0.0061(3)$ & $0.0109(3)$ & $0.0026(3)$ & $0.0043(3)$ & $-0.0012(3)$ \\
O4 & $0.0057(3)$ & $0.0080(3)$ & $0.0124(3)$ & $-0.0002(3)$ & $0.0037(3)$ & $0.0002(3)$ \\
O5 & $0.0063(3)$ & $0.0081(3)$ & $0.0124(3)$ & $-0.0022(3)$ & $0.0024(3)$ & $0.0024(3)$ \\
O6 & $0.0065(3)$ & $0.0089(3)$ & $0.0047(3)$ & $-0.0008(3)$ & $0.0019(2)$ & $-0.0019(2)$ \\
& & & & & & \\
\hline
\end{tabular}


Geometric parameters $\left(\AA,{ }^{\circ}\right)$

\begin{tabular}{|c|c|c|c|}
\hline $\mathrm{Fe} 1-\mathrm{O}^{\mathrm{i}}$ & $1.9456(9)$ & $\mathrm{P} 2-\mathrm{O} 6^{\mathrm{v}}$ & $1.5468(8)$ \\
\hline $\mathrm{Fe} 1-\mathrm{O}^{\mathrm{i}}$ & $2.0158(9)$ & $\mathrm{P} 2-\mathrm{O} 6$ & $1.5468(8)$ \\
\hline $\mathrm{Fe} 1-\mathrm{O} 2^{\mathrm{ii}}$ & $2.0374(9)$ & $\mathrm{Na} 1-\mathrm{O} 5^{\mathrm{iii}}$ & $2.2895(9)$ \\
\hline $\mathrm{Fe} 1-\mathrm{O}^{\mathrm{iii}}$ & $2.0543(9)$ & $\mathrm{Na} 1-\mathrm{O} 5$ & $2.2895(9)$ \\
\hline $\mathrm{Fe} 1-\mathrm{O} 1^{\mathrm{iv}}$ & $2.0724(8)$ & $\mathrm{Na} 1-\mathrm{O} 4^{\mathrm{iii}}$ & $2.3835(9)$ \\
\hline $\mathrm{Fe} 1-\mathrm{O} 1$ & $2.1434(9)$ & $\mathrm{Na} 1-\mathrm{O} 4$ & $2.3836(9)$ \\
\hline $\mathrm{Co} 2-\mathrm{O}_{4}^{v}$ & $2.1072(9)$ & $\mathrm{Na} 1-\mathrm{O} 4^{\text {viii }}$ & $2.5217(9)$ \\
\hline $\mathrm{Co} 2-\mathrm{O} 4$ & $2.1072(9)$ & $\mathrm{Na} 1-\mathrm{O} 4^{\mathrm{v}}$ & $2.5218(9)$ \\
\hline $\mathrm{Co} 2-\mathrm{O} 2^{\mathrm{ii}}$ & $2.1567(9)$ & $\mathrm{Na} 1-\mathrm{O}^{\mathrm{v}}$ & $2.8754(10)$ \\
\hline $\mathrm{Co} 2-\mathrm{O} 2^{\mathrm{vi}}$ & $2.1568(9)$ & $\mathrm{Na} 1-\mathrm{O} 5^{\text {viii }}$ & $2.8754(10)$ \\
\hline $\mathrm{Co} 2-\mathrm{O}^{\mathrm{vii}}$ & $2.1632(8)$ & $\mathrm{Na} 2-\mathrm{O} 3^{\text {ix }}$ & $2.3940(9)$ \\
\hline $\mathrm{Co} 2-\mathrm{O}^{\mathrm{iii}}$ & $2.1632(8)$ & $\mathrm{Na} 2-\mathrm{O} 3$ & $2.3940(9)$ \\
\hline $\mathrm{P} 1-\mathrm{O} 4$ & $1.5206(9)$ & $\mathrm{Na} 2-\mathrm{O} 3^{\mathrm{x}}$ & $2.5269(10)$ \\
\hline $\mathrm{P} 1-\mathrm{O} 3$ & $1.5336(9)$ & $\mathrm{Na} 2-\mathrm{O} 3^{\mathrm{vii}}$ & $2.5269(10)$ \\
\hline $\mathrm{P} 1-\mathrm{O} 1$ & $1.5422(9)$ & $\mathrm{Na} 2-\mathrm{O} 2^{\text {ix }}$ & $2.8158(15)$ \\
\hline $\mathrm{P} 1-\mathrm{O} 2$ & $1.5502(9)$ & $\mathrm{Na} 2-\mathrm{O} 2$ & $2.8158(15)$ \\
\hline $\mathrm{P} 2-\mathrm{O} 5^{\mathrm{v}}$ & $1.5240(9)$ & $\mathrm{Na} 2-\mathrm{O} 6^{\mathrm{xi}}$ & $2.8513(16)$ \\
\hline $\mathrm{P} 2-\mathrm{O} 5$ & $1.5241(9)$ & $\mathrm{Na} 2-\mathrm{O} 6^{\mathrm{xii}}$ & $2.8513(16)$ \\
\hline $\mathrm{O} 5^{\mathrm{i}}-\mathrm{Fe} 1-\mathrm{O} 3^{\mathrm{i}}$ & $94.91(4)$ & $\mathrm{O} 5-\mathrm{Na} 1-\mathrm{O} 4^{\text {viii }}$ & $73.59(3)$ \\
\hline $\mathrm{O} 5^{\mathrm{i}}-\mathrm{Fe} 1-\mathrm{O} 2^{\mathrm{ii}}$ & $110.51(4)$ & $\mathrm{O} 4^{\mathrm{iii}}-\mathrm{Na} 1-\mathrm{O} 4^{\text {viii }}$ & $67.32(4)$ \\
\hline $\mathrm{O} 3^{\mathrm{i}}-\mathrm{Fe} 1-\mathrm{O} 2^{\mathrm{ii}}$ & $86.18(4)$ & $\mathrm{O} 4-\mathrm{Na} 1-\mathrm{O} 4^{\mathrm{viii}}$ & $112.68(4)$ \\
\hline $\mathrm{O} 5^{\mathrm{i}}-\mathrm{Fe} 1-\mathrm{O}^{\mathrm{iii}}$ & $164.12(4)$ & 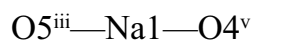 & $73.59(3)$ \\
\hline $\mathrm{O} 3^{\mathrm{i}}-\mathrm{Fe} 1-\mathrm{O}^{\mathrm{iii}}$ & $98.29(4)$ & $\mathrm{O} 5-\mathrm{Na} 1-\mathrm{O} 4^{\mathrm{v}}$ & $106.41(3)$ \\
\hline $\mathrm{O} 2^{\mathrm{ii}}-\mathrm{Fe} 1-\mathrm{O}^{\mathrm{iii}}$ & $79.27(3)$ & 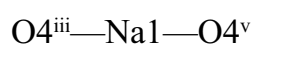 & $112.68(4)$ \\
\hline $\mathrm{O} 5^{\mathrm{i}}-\mathrm{Fe} 1-\mathrm{O} 1^{\mathrm{iv}}$ & $86.97(4)$ & $\mathrm{O} 4-\mathrm{Na} 1-\mathrm{O} 4^{\mathrm{v}}$ & $67.32(4)$ \\
\hline $\mathrm{O} 3^{\mathrm{i}}-\mathrm{Fe} 1-\mathrm{O} 1^{\mathrm{iv}}$ & $99.56(4)$ & $\mathrm{O} 4^{\mathrm{viii}}-\mathrm{Na} 1-\mathrm{O} 4^{\mathrm{v}}$ & 180.0 \\
\hline $\mathrm{O} 2^{\mathrm{ii}}-\mathrm{Fe} 1-\mathrm{O} 1^{\mathrm{iv}}$ & $161.21(4)$ & $\mathrm{O} 5^{\mathrm{iii}}-\mathrm{Na} 1-\mathrm{O}^{\mathrm{v}}$ & $126.25(4)$ \\
\hline $\mathrm{O} 6^{\mathrm{iii}}-\mathrm{Fe} 1-\mathrm{O} 1^{\mathrm{iv}}$ & $82.19(3)$ & $\mathrm{O} 5-\mathrm{Na} 1-\mathrm{O}^{\mathrm{v}}$ & $53.75(4)$ \\
\hline 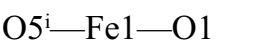 & $81.40(4)$ & $\mathrm{O} 4^{\mathrm{iii}}-\mathrm{Na} 1-\mathrm{O}^{\mathrm{v}}$ & $86.18(3)$ \\
\hline $\mathrm{O} 3^{\mathrm{i}}-\mathrm{Fe} 1-\mathrm{O} 1$ & $174.04(4)$ & $\mathrm{O} 4-\mathrm{Na} 1-\mathrm{O}^{\mathrm{v}}$ & $93.82(3)$ \\
\hline $\mathrm{O} 22^{\mathrm{ii}}-\mathrm{Fe} 1-\mathrm{O} 1$ & $90.73(3)$ & $\mathrm{O} 4^{\mathrm{viii}}-\mathrm{Na} 1-\mathrm{O}^{\mathrm{v}}$ & $114.14(3)$ \\
\hline $\mathrm{O}^{\mathrm{iii}}-\mathrm{Fe} 1-\mathrm{O} 1$ & $86.11(3)$ & $\mathrm{O} 4^{\mathrm{v}}-\mathrm{Na} 1-\mathrm{O} 5^{\mathrm{v}}$ & $65.86(3)$ \\
\hline $\mathrm{O} 1{ }^{\mathrm{iv}}-\mathrm{Fe} 1-\mathrm{O} 1$ & $84.97(3)$ & $\mathrm{O} 5^{\mathrm{iii}}-\mathrm{Na} 1-\mathrm{O} 5^{\mathrm{viii}}$ & $53.75(4)$ \\
\hline $\mathrm{O} 4 \mathrm{v}-\mathrm{Co} 2-\mathrm{O} 4$ & $80.44(5)$ & $\mathrm{O} 5-\mathrm{Na} 1-\mathrm{O} 5^{\text {viii }}$ & $126.25(4)$ \\
\hline $\mathrm{O} 4^{\mathrm{v}}-\mathrm{Co} 2-\mathrm{O} 2^{\mathrm{ii}}$ & $165.05(3)$ & $\mathrm{O} 4^{\mathrm{iii}}-\mathrm{Na} 1-\mathrm{O} 5^{\mathrm{viii}}$ & $93.82(3)$ \\
\hline $\mathrm{O} 4-\mathrm{Co} 2-\mathrm{O} 2^{\mathrm{ii}}$ & $86.54(3)$ & $\mathrm{O} 4-\mathrm{Na} 1-\mathrm{O} 5^{\text {viii }}$ & $86.18(3)$ \\
\hline $\mathrm{O} 4^{\mathrm{v}}-\mathrm{Co} 2-\mathrm{O} 2^{\mathrm{vi}}$ & $86.54(3)$ & 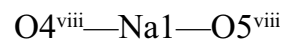 & $65.86(3)$ \\
\hline $\mathrm{O} 4-\mathrm{Co} 2-\mathrm{O} 2^{\mathrm{vi}}$ & $165.05(3)$ & $\mathrm{O} 4^{\mathrm{v}}-\mathrm{Na} 1-\mathrm{O} 5^{\mathrm{viii}}$ & $114.14(3)$ \\
\hline $\mathrm{O} 2^{\mathrm{ii}}-\mathrm{Co} 2-\mathrm{O} 2^{\mathrm{vi}}$ & $107.25(5)$ & $\mathrm{O} 5^{\mathrm{v}}-\mathrm{Na} 1-\mathrm{O} 5^{\mathrm{viii}}$ & 180.0 \\
\hline $\mathrm{O} 4^{\mathrm{v}}-\mathrm{Co} 2-\mathrm{O} 6^{\mathrm{vii}}$ & $92.44(3)$ & $\mathrm{O} 3^{\mathrm{ix}}-\mathrm{Na} 2-\mathrm{O} 3$ & $172.39(8)$ \\
\hline $\mathrm{O} 4-\mathrm{Co} 2-\mathrm{O}^{\mathrm{vii}}$ & $113.30(3)$ & $\mathrm{O} 3^{\mathrm{ix}}-\mathrm{Na} 2-\mathrm{O} 3^{\mathrm{x}}$ & $81.52(3)$ \\
\hline $\mathrm{O} 2^{\mathrm{ii}}-\mathrm{Co} 2-\mathrm{O}^{\mathrm{vii}}$ & $85.96(3)$ & $\mathrm{O} 3-\mathrm{Na} 2-\mathrm{O} 3^{\mathrm{x}}$ & $97.99(3)$ \\
\hline $\mathrm{O} 2^{\mathrm{vi}}-\mathrm{Co} 2-\mathrm{O}^{\mathrm{vii}}$ & $74.34(3)$ & $\mathrm{O} 3^{\text {ix }}-\mathrm{Na} 2-\mathrm{O} 3^{\text {vii }}$ & $97.99(3)$ \\
\hline $\mathrm{O} 4^{\mathrm{v}}-\mathrm{Co} 2-\mathrm{O} 6^{\mathrm{iii}}$ & $113.30(3)$ & $\mathrm{O} 3-\mathrm{Na} 2-\mathrm{O} 3^{\mathrm{vii}}$ & $81.52(3)$ \\
\hline $\mathrm{O} 4-\mathrm{Co} 2-\mathrm{O}^{\mathrm{iii}}$ & $92.44(3)$ & $\mathrm{O} 3^{\mathrm{x}}-\mathrm{Na} 2-\mathrm{O} 3^{\mathrm{vii}}$ & $172.68(8)$ \\
\hline
\end{tabular}




\begin{tabular}{|c|}
\hline $\mathrm{O} 2^{\mathrm{ii}}-\mathrm{Co} 2-\mathrm{O}^{\mathrm{iii}}$ \\
\hline $\mathrm{O} 2^{\mathrm{vi}}-\mathrm{Co} 2-\mathrm{O} 6^{\mathrm{iii}}$ \\
\hline $\mathrm{O}^{\mathrm{vii}}-\mathrm{Co} 2-\mathrm{O}^{\mathrm{iii}}$ \\
\hline $\mathrm{O} 4-\mathrm{P} 1-\mathrm{O} 3$ \\
\hline $\mathrm{O} 4-\mathrm{P} 1-\mathrm{O} 1$ \\
\hline $\mathrm{O} 3-\mathrm{P} 1-\mathrm{O} 1$ \\
\hline $\mathrm{O} 4-\mathrm{P} 1-\mathrm{O} 2$ \\
\hline $\mathrm{O} 3-\mathrm{P} 1-\mathrm{O} 2$ \\
\hline $\mathrm{O} 1-\mathrm{P} 1-\mathrm{O} 2$ \\
\hline $\mathrm{O} 5^{\mathrm{v}}-\mathrm{P} 2-\mathrm{O} 5$ \\
\hline $\mathrm{O} 5^{\mathrm{v}}-\mathrm{P} 2-\mathrm{O}^{\mathrm{v}}$ \\
\hline $\mathrm{O} 5-\mathrm{P} 2-\mathrm{O}^{\mathrm{v}}$ \\
\hline $\mathrm{O} 5^{\mathrm{v}}-\mathrm{P} 2-\mathrm{O} 6$ \\
\hline $\mathrm{O} 5-\mathrm{P} 2-\mathrm{O} 6$ \\
\hline $\mathrm{O} 6^{\mathrm{v}}-\mathrm{P} 2-\mathrm{O} 6$ \\
\hline $\mathrm{O} 5^{\mathrm{iii}}-\mathrm{Na} 1-\mathrm{O} 5$ \\
\hline 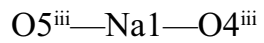 \\
\hline $\mathrm{O} 5-\mathrm{Na} 1-\mathrm{O} 4^{\mathrm{iii}}$ \\
\hline 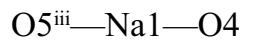 \\
\hline $\mathrm{O} 5-\mathrm{Na} 1-\mathrm{O} 4$ \\
\hline $\mathrm{O} 4{ }^{\mathrm{iii}}-\mathrm{Na} 1-\mathrm{O} 4$ \\
\hline $\mathrm{O} 5^{\mathrm{iii}-\mathrm{Na} 1-\mathrm{O}}$ \\
\hline
\end{tabular}

$74.34(3)$

$85.96(3)$

$146.65(5)$

$112.98(5)$

$108.59(5)$

$108.95(5)$

$110.92(5)$

$106.53(5)$

$108.78(5)$

$103.42(7)$

$108.79(5)$

$112.97(5)$

$112.97(5)$

$108.79(5)$

$109.82(7)$

180.0

78.23 (3)

101.77 (3)

101.77 (3)

$78.23(3)$

180.0

$106.41(3)$

$$
\begin{aligned}
& \mathrm{O} 3^{\mathrm{ix}}-\mathrm{Na} 2-\mathrm{O} 2^{\text {ix }} \\
& \mathrm{O} 3-\mathrm{Na} 2-\mathrm{O} 2^{\mathrm{ix}} \\
& \mathrm{O} 3^{\mathrm{x}}-\mathrm{Na} 2-\mathrm{O}^{\mathrm{ix}}
\end{aligned}
$$

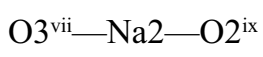

$$
\begin{aligned}
& \mathrm{O} 3{ }^{\mathrm{ix}}-\mathrm{Na} 2-\mathrm{O} 2 \\
& \mathrm{O} 3-\mathrm{Na} 2-\mathrm{O} 2 \\
& \mathrm{O} 3{ }^{\mathrm{x}}-\mathrm{Na} 2-\mathrm{O} 2 \\
& \mathrm{O} 3{ }^{\text {vii- }-\mathrm{Na} 2-\mathrm{O} 2} \\
& \mathrm{O} 2{ }^{\mathrm{ix}}-\mathrm{Na} 2-\mathrm{O} 2 \\
& \mathrm{O} 3^{\mathrm{ix}}-\mathrm{Na} 2-\mathrm{O}^{\mathrm{xi}} \\
& \mathrm{O} 3-\mathrm{Na} 2-\mathrm{O}^{\mathrm{xi}} \\
& \mathrm{O} 3^{\mathrm{x}}-\mathrm{Na} 2-\mathrm{O}^{\mathrm{xi}}
\end{aligned}
$$

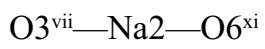

$$
\begin{aligned}
& \mathrm{O} 2^{\mathrm{ix}}-\mathrm{Na} 2-\mathrm{O} 6^{\mathrm{xi}} \\
& \mathrm{O} 2-\mathrm{Na} 2-\mathrm{O}^{\mathrm{xi}} \\
& \mathrm{O}^{\mathrm{ix}}-\mathrm{Na} 2-\mathrm{O}^{\mathrm{xii}} \\
& \mathrm{O} 3-\mathrm{Na} 2-\mathrm{O}^{\mathrm{xii}} \\
& \mathrm{O} 3^{\mathrm{x}}-\mathrm{Na} 2-\mathrm{O}^{\mathrm{xii}}
\end{aligned}
$$

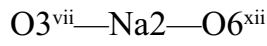

$$
\begin{aligned}
& \mathrm{O} 2^{\mathrm{ix}}-\mathrm{Na} 2-\mathrm{O}^{\mathrm{xii}} \\
& \mathrm{O} 2-\mathrm{Na} 2-\mathrm{O}^{\mathrm{xii}} \\
& \mathrm{O} 6^{\mathrm{xi}}-\mathrm{Na} 2-\mathrm{O}^{\mathrm{xii}}
\end{aligned}
$$

$55.93(3)$

$117.11(5)$

$62.15(3)$

$111.51(5)$

$117.11(5)$

$55.93(3)$

$111.51(5)$

$62.15(3)$

$76.15(5)$

$116.10(5)$

$71.28(4)$

83.54 (4)

103.11 (4)

145.12 (3)

$126.19(2)$

71.28 (4)

$116.10(5)$

103.11 (4)

83.54 (4)

126.19 (2)

145.12 (3)

52.71 (4)

Symmetry codes: (i) $-x+3 / 2, y+1 / 2,-z+1 / 2$; (ii) $-x+3 / 2,-y+3 / 2,-z+1$; (iii) $-x+1,-y+1,-z$; (iv) $-x+3 / 2,-y+3 / 2,-z$; (v) $-x+1, y,-z+1 / 2$; (vi) $x-1 / 2$, $-y+3 / 2, z-1 / 2$; (vii) $x,-y+1, z+1 / 2$; (viii) $x,-y+1, z-1 / 2$; (ix) $-x+2, y,-z+3 / 2$; (x) $-x+2,-y+1,-z+1$; (xi) $x+1 / 2,-y+1 / 2, z+1 / 2 ;(x i i)-x+3 / 2,-y+1 / 2$, $-z+1$. 